\title{
Plasmid sonication improves sequencing efficiency and quality in the Beckman Coulter CEQ system
}

\author{
Mark D. Thompson', Kelly G. Aukema², Dana M. O'Bryan', Stephen D. \\ Rader $^{2}$, and Brent W. Murray ${ }^{3}$ \\ ${ }^{1}$ College of Science and Management, 2 Department of Chemistry, and ${ }^{3}$ Ecosystem \\ Science and Management Program, University of Northern British Columbia, Prince \\ George, British Columbia, Canada
}

BioTechniques 45:327-329 (September 2008)

doi 10.2144/000112902

We report on an unexpectedly high rate of unreadable chromatograms from plasmid sequencing using Beckman Coulter's protocols, chemistry, and CEQ8000 instrument. Failed or poor quality plasmid sequence chromatograms were accompanied by a sharp drop, fluctuation, or steady decline in the current and a corresponding delay in signal counts beyond the time of capillary injection. We observe a correlation between the presence of supercoiled DNA and these sequencing problems. Herein we demonstrate that plasmid sonication, which is known to fragment supercoiled DNA, is an effective way to improve sequence phred20 read lengths to the point that they are not significantly different from Beckman Coulter's control template or enzymatically linearized plasmids.

Numerous attempts by our facility to sequence a diversity of plasmids using Beckman Coulter's (BC; Fullerton, CA, USA) dye terminator cycle sequencing (DTCS) and the CEQ8000 system produced an unexpectedly high rate of unreadable sequence chromatograms. The raw data signals for many failed or poor quality plasmid sequences were accompanied by either a drop or anomaly in the current and a delay in signal beyond the $20 \mathrm{~min}$ injection point. We hypothesize that these results are caused by the presence of supercoiled DNA, which may interfere with the sequencing reaction and/or hinder electrophoretic mobility.

All forms of covalently closed circular DNA (ccDNA) exhibit some measure of supercoiling caused by twist, writhe, and excess turns $(1,2)$. Solvent chemistry and temperature affect the physics of ccDNA isomers to form different supercoiled topologies, or topoisomers, that relax when one or both strands are nicked (3). Topoisomers and relaxed forms of ccDNA are distinguished by their unique electrophoretic mobility characteristics (4).

The pre-heat treatments prescribed by BC for plasmid DTCS are said to denature plasmid for improved signal strength and current stability. Although $\mathrm{BC}$ protocols prescribe three different pre-heat treatments for "certain plasmid
DNA templates," we observed no significant difference in sequence quality between the three pre-heat $(\mathrm{PH})$ treatments. Plasmids are generally resistant to cleavage at $95^{\circ} \mathrm{C}$, but rapidly degrade when temperatures reach $107^{\circ} \mathrm{C}(5)$. Unless PH treatments nick one or both strands of ccDNA into relaxed form, the denatured state will not necessarily be retained and topoisomers will be carried forward into both the cycle sequencing reaction and capillary injection phases.

Using prescribed $\mathrm{BC}$ methods, we report an average phred20 read length of $127 \mathrm{bp}$ for readable sequences and complete sequencing failure for $27 \%$ of plasmids purified with the Qiagen QIAprep Spin Miniprep kit (Mississauga, ON, Canada). In contrast, we report $>95 \%$ success rate for BC-supplied pUC18 control plasmid. Agarose gel electrophoresis reveals a higher ratio of nicked to supercoiled topoisomer in the BC-supplied pUC18 compared with pUC18 prepared with the Qiagen Miniprep kit (abbreviated pUC18-Q herein; Figure 1, lanes 1 and 2 ). To test whether the presence of a high ratio of supercoiled topoisomers leads to the reported sequencing problems, we digested plasmids with restriction enzymes prior to sequencing (Figure 1, lane 3). Restriction digestion (RD) improved both read length and current values that are equivalent with pUC18 control and linear template sequencing. While RD improves sequencing quality, this is not a practical solution due to the extra expense, time, and handling, which, importantly, are not needed for other manufacturers' sequencing systems.

We investigated sonication as an alternative method. Sonication is used for nicking and shearing DNA in large-scale shotgun sequencing projects and for the construction of microsatellite libraries $(6,7)$. Empirically, we determined that sonication of PH-treated plasmids for $2 \mathrm{~s}$ at power setting 2 (abbreviated PHS herein) using a sonic dismembrator (Model 100; Fisher Scientific, Ottawa, ON, Canada) fragments most supercoiled DNA (Figure 1, lane 4).

To assess the quality of our sequencing results from a variety of plasmids (Table 1), we compiled phred20 scores (8) for 386 sequence trace files generated from plasmid templates by our sequencing facility from August 2005 until November 2007. During this time period, plasmids received one of the three treatments (PH, $n=125$; PHS, $n=171 ; \mathrm{RD}, n=90)$ prior to sequencing. The pUC18 control template provided by $\mathrm{BC}(n=23)$ received no treatment. The BC PH treatment shows significantly smaller mean phred20 scores compared with PHS, RD, or the pUC18 control (Figure 2).

Our results clearly show that PHS or $\mathrm{RD}$ of supercoiled topoisomers eliminate our plasmid sequencing problems. Current anomalies are observed during electrophoresis when sequencing both untreated plasmids, other than the

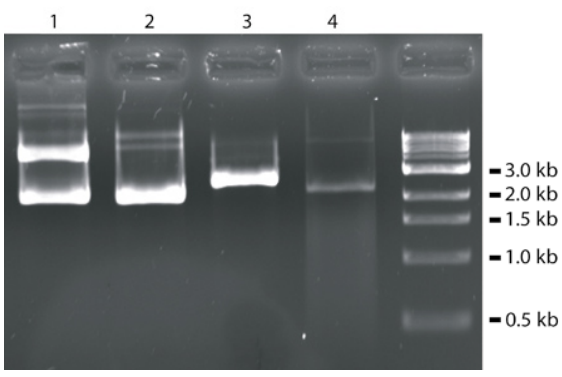

Figure 1. Sonication efficiently linearizes plasmid DNA. Equal molar amounts of pUC18 plasmid DNA from two sources were electrophoresed on a $1 \%$ agarose gel: control pUC18 from Beckman Coulter (untreated, lane 1) and pUC18 prepared with a Qiagen QIAprep Spin Miniprep kit (untreated, lane 2; EcoRI-digested, lane 3; and sonicated, lane 4). Supercoiled forms are observed at $\sim 2 \mathrm{~kb}$ in lanes 1,2 , and 4 . A bright nicked form is observed in lane $1 \mathrm{at} \sim 5 \mathrm{~kb}$. 


\section{Benchmarks}

pUC18 control, and PCR templates contaminated with plasmids through colony PCR (9); anomalies are more pronounced (e.g., Figure 2, lower panel $[\mathrm{PH}]$ ) for larger plasmids. Coupled with a delay in fluorescent signal counts, these anomalies support the hypothesis that supercoiled topoisomers inhibit electrophoretic mobility through the capillary. We tested this hypothesis further by adding supercoiled plasmid to $\mathrm{BC}$ pUC18 control reactions. Completed cycle sequencing reactions $(n=8)$ were each divided into three; one control and two treatments, to which were added $50 \mathrm{fmol}$ of pUC18-Q (3 $\mathrm{kb})$ or pCR-TOPO $(5 \mathrm{~kb})$ plasmid. Paired $t$-tests showed that only the addition of the larger pCR-TOPO plasmid caused a significant reduction in phred20 scores (Mean difference $=50.2, P=0.002)$. Although current fluctuations were not observed in this experiment, similar tests with the pCR-TOPO and similar-sized vectors showed current abnormalities in $43 \%$ of plasmid additions $(n=13)$.

Template accessibility during the sequencing reaction may also be improved by relieving supercoiling. As noted, the addition of pUC18-Q plasmids to sequenced BC pUC18 controls does not significantly affect read length. Sequencing the pUC18-Q plasmid with only a PH treatment (i.e., not PHS or RD), however, leads to significantly lower phred20 scores $($ Mean $=209, P=0.001)$ coupled with peak resolution problems (irregular spacing and peak shape, and a high background), which are also observed when sequencing other PH-treated
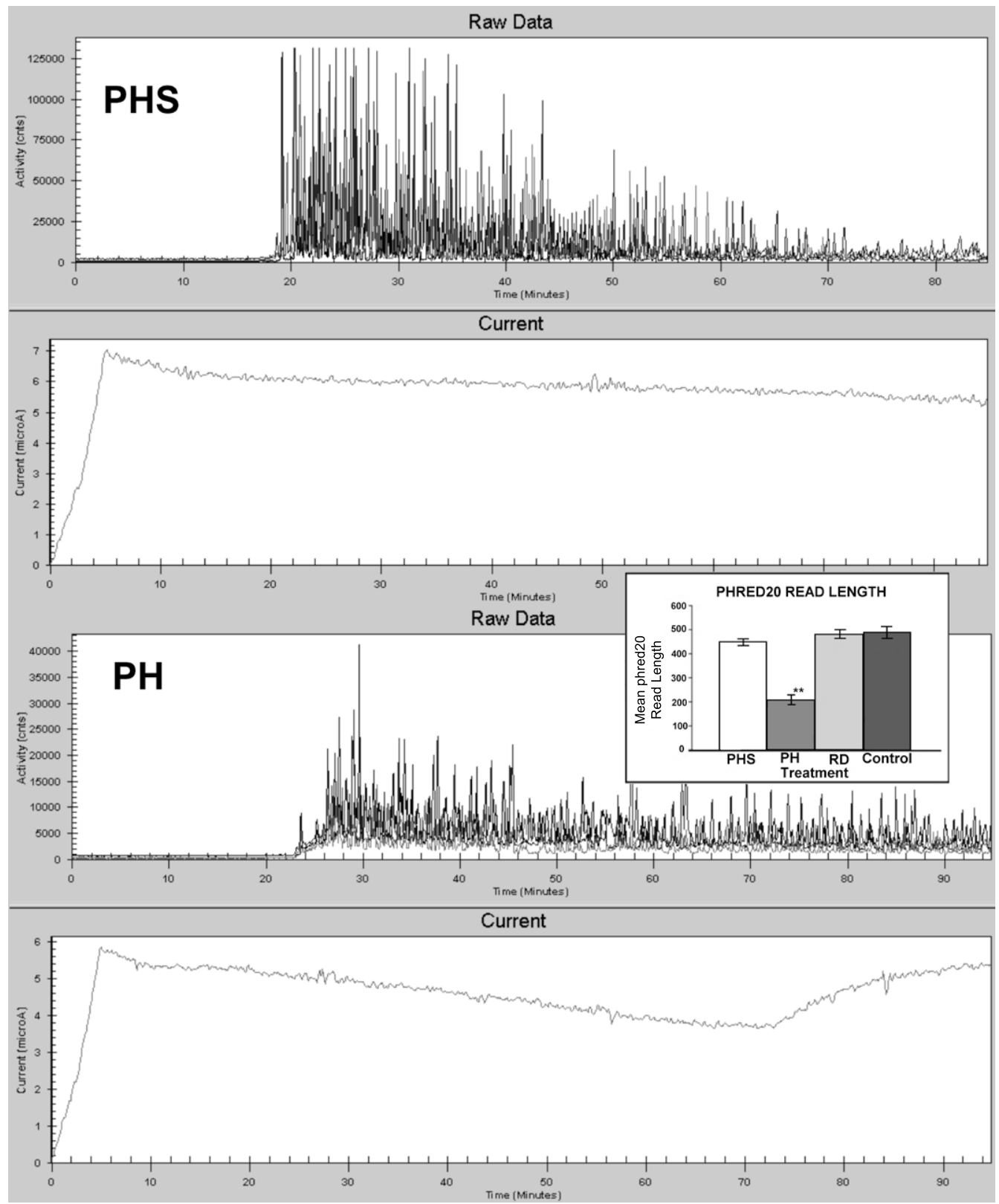

Figure 2. Template sonication results in significantly longer sequence read lengths. Two representative sequence runs showing raw fluorescent signal and current levels for plasmid receiving the pre-heat plus sonication (PHS) treatment (PHS, upper panel) and the pre-heat $(\mathrm{PH})$ treatment (lower panel). A time delay in raw data signal to $33 \mathrm{~min}$ corresponds with a marked drop in current levels. The inset shows a histogram with standard error bars and a significant increase [GT2 test $(10), P<0.01 * *]$ in mean phred20 read length (failed sequences were not included in these calculations) in all methods compared with the PH treatment; control refers to the Beckman Coulter (BC) pUC18 control sequence.

plasmids. In contrast, the addition of larger plasmids is associated with reduced read lengths and current anomalies, however, it does not affect peak shapes, spacing, or levels of background. We speculate that a high ratio of supercoiled plasmid also interferes with DTCS efficiency, causing peak resolution problems.

The improvements to sequencing plasmids that we report herein will have immediate benefits for researchers using CEQ instruments. Although extreme care is required to obtain high ratios of supercoiled DNA using the traditional plasmid mini-prep technique (6), plasmid DNA obtained from the Qiagen Miniprep kit consistently yields the vast majority of the plasmid in the undamaged supercoiled form (Figure 1). Other commercially available 
Table 1. Plasmid Types and Sizes (Including Inserts)

\begin{tabular}{|ll|}
\hline Plasmid & Size (kb) \\
\hline pGEM-T & 3.3 \\
pUC18 & $2.7-4.7$ \\
pUC19 & $3-4$ \\
pBluescript & $3.5-4.1$ \\
pET3 & 5 \\
pET3a & $5-6$ \\
pET21b & $6-7.3$ \\
pET-34b(+) & 5.9 \\
\hline pET-AK (pET-15b) & $5.2-7.1$ \\
pACYC-DUET1 & $4-5.2$ \\
pCR 2.1 TOPO & 5 \\
\hline pENTR & 4.5 \\
pSE358 & 5.5 \\
pSE362 & 5.8 \\
pSE363 & 6.5 \\
\hline pRS404 & 7.5 \\
\hline The above plasmid types have been se- \\
quenced in our facility.All were prepared using \\
Qiagen QIAprep Spin Miniprep kits. \\
\hline
\end{tabular}

plasmid extraction kits use silica membrane technology (e.g., Promega's "Wizard," Invitrogen's "Purelink," and Mo Bio's "UltraClean") optimized for yielding high ratios of supercoiled DNA. Extraction kits are standard in most molecular genetic laboratories and are the basis of automation techniques. The PHS is a simple, fast, and costeffective method that can easily be incorporated into any laboratory. Removing the supercoiled structure improves sequencing efficiency while ensuring optimal phred 20 read lengths.

\section{ACKNOWLEDGEMENTS}

This analysis was made possible through the kind donation of sequence traces that were generated for other scientific studies in the labs of B.W.M., S.D.R., Andrea Gorrell, and Chow Lee. This work was supported in part by National Sciences and Engineering Research Council of Canada (NSERC) Discovery Grant 298521 and a Research Corporation CCS Award to S.D.R. and by NSERC Discovery Grant 249958 to B.W.M. This work was also made possible through funding from the University of Northern British Columbia College of Science of Management and a Michael
Smith Foundation postdoctoral fellowship to K.G.A.

\section{COMPETING INTERESTS STATEMENT}

The authors declare no competing interests.

\section{REFERENCES}

1. Fogg, J.M., N. Kolmakova, I. Rees, S. Magonov, H. Hansma, J.J. Perona, and E.L. Zechiedrich. 2006. Exploring writhe in supercoiled minicircle DNA. J. Phys.: Condens. Matter 18:S145-S159.

2. Frank-Kamenetskii, M.D. 1997. Biophysics of the DNA molecule. Phys. Rep. 288:13-60.

3. Tweedie, J.W. and M.S. Stowell. 2005. Quantification of DNA by agarose gel electrophoresis and analysis of the topoisomers of plasmid and M13 DNA following treatment with a restriction endonuclease or DNA topoisomerase I. Biochem. Mol. Biol. Educ. 33:28-33.

4. Viglasky, V., M. Antalik, J. Adamcik, and D. Podhradsky. 2000. Early melting of supercoiled DNA topoisomers observed by TGGE. Nucleic Acids Res. 28:e51.

5. Marguet, E. and P. Forterre. 1998. Protection of DNA by salts against thermodegradation at temperatures typical for hyperthermophiles. Extremophiles 2:115-122.

6. Sambrook, J. and D.W. Russell. 2001. Molecular Cloning: A Laboratory Manual, 3rd ed. CSH Laboratory Press, Cold Spring Harbor, NY.

7. Zane, L., L. Bargelloni, and T. Patarnello. 2002. Strategies for microsatellite isolation: a review. Mol. Ecol. 11:1-16.

8. Ewing, B., L. Hillier, M.C. Wendl, and P. Green. 1998. Base-calling of automated sequencer traces using phred. I. Accuracy assessment. Genome Res. 8:175-185.

9. Menossi, M., N. Cremonese, L.G. Maron, and P. Arruda. 2000. Making colony PCR easier by adding gel-loading buffer to the amplification reaction. BioTechniques 28:424-426.

10. Sokal, R.R. and F.J. Rohlf. 1995. Biometry: the Principles and Practice of Statistics in Biological Research. W. H. Freeman and Company, New York.

Received 17 December 2007; accepted 28 May 2008.

Address correspondence to Brent Murray, University of Northern British Columbia, Ecosystem Science and Management Program, Prince George, BC, V2N 4Z9, Canada.e-mail:murrayb@unbc.ca

To purchase reprints of this article, contact: Reprints@BioTechniques.com

\section{BioTechniques}

Connecting. Informing. Advancing. For 25 Years.

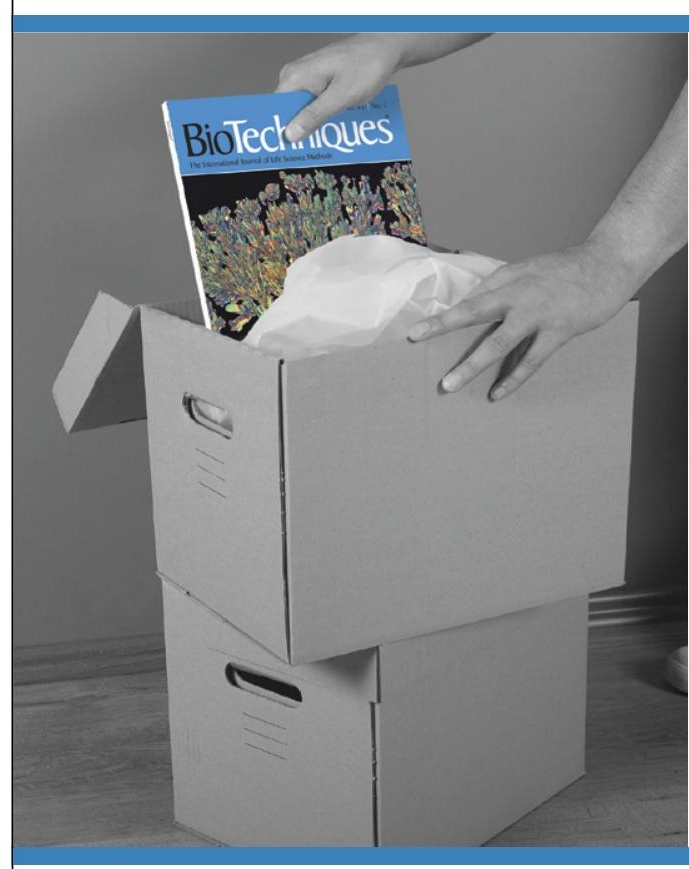

\section{Update your subscription}

Update your profile today to ensure that your copy of BioTechniques experiences no delivery disruptions.

Go to:

www.biotechniques.com/subscribe 